\title{
The Role of Peroxiredoxin Family in Cancer Signaling
}

REVIEW

\author{
Yosup Kim ${ }^{1}$, Ho Hee Jang ${ }^{1,2}$ \\ ${ }^{1}$ Department of Health Sciences and Technology, Graduate School of Medicine, Gachon University, ${ }^{2}$ Department of Biochemistry, College of \\ Medicine, Gachon University, Incheon, Korea
}

\begin{abstract}
Peroxiredoxins (Prxs) are antioxidant enzymes that protect cells from oxidative stress by reducing intracellular accumulation of reactive oxygen species (ROS). In mammalian cells, the six Prx isoforms are ubiquitously expressed in diverse intracellular locations. They are involved in the regulation of various physiological processes including cell growth, differentiation, apoptosis, immune response and metabolism as well as intracellular ROS homeostasis. Although there are increasing evidences that Prxs are involved in carcinogenesis of many cancers, their role in cancer is controversial. The ROS levels in cancer cells are increased compared to normal cells, thus promoting cancer development. Nevertheless, for various cancer types, an overexpression of Prxs has been found to be associated with poor patient prognosis, and an increasing number of studies have reported that tumorigenesis is either facilitated or inhibited by regulation of cancer-associated signaling pathways. This review summarizes Prx isoforms and their basic functions, the relationship between the expression level and the physiological role of Prxs in cancer cells, and their roles in regulating cancer-associated signaling pathways.
\end{abstract}

(J Cancer Prev 2019;24:65-71)

Key Words: Peroxiredoxins, Oxidative stress, Cancer, Peroxidase activity, Tumorigenesis

\section{INTRODUCTION}

Cancer is a heterogeneous disease caused by multiple and complex risk factors, and it poses a substantial threat to the quality of human life [1]. An increase in reactive oxygen species (ROS) from endogenous or exogenous sources induces intracellular oxidative stress, causing various diseases. It has also been increasingly reported to promote tumorigenesis, including the proliferation, invasion, and metastasis of cancer cells [2,3]. Healthy cells have diverse anti-oxidative defense mechanisms to maintain ROS homeostasis, and enzymatic and non-enzymatic antioxidants provide the most effective system for cellular protection against ROS-driven oxidative stress by removing intracellular ROS [4,5].

Peroxiredoxins (Prxs) represent one of the diverse enzymatic antioxidant systems that are distributed across various organelles, and different subtypes of Prxs are stratified according to the number and the position of Cys residues [6,7]. Prxs are known to either facilitate or inhibit tumorigenesis, depending on the cancer type, by regulating the ROS level [8]. Furthermore, an increasing number of studies have reported that, in addition to the peroxidase function that removes hydrogen peroxide $\left(\mathrm{H}_{2} \mathrm{O}_{2}\right)$, Prxs regulate cancer signaling pathways in a redox-dependent or -independent manner through interaction with other signaling proteins [9-12]. This review focuses on the physiological roles of Prx isoforms in cancer and the mechanism behind their ability to regulate signaling pathways by interacting with target proteins. Lastly, this review discusses the potential of Prxs as novel therapeutic targets.

Received June 18, 2019, Revised June 22, 2019, Accepted June 24, 2019

Correspondence to: Ho Hee Jang

E-mail: hhjang@gachon.ac.kr, ORCID: Ho Hee Jang, https://orcid.org/0000-0003-0314-8230

Copyright (C) 2019 Korean Society of Cancer Prevention

(c) This is an Open Access article distributed under the terms of the Creative Commons Attribution Non-Commercial License (http://creativecommons.org/licenses/by-nc/4.0) which permits unrestricted non-commercial use, distribution, and reproduction in any medium, provided the original work is properly cited. 


\section{PEROXIREDOXIN ISOFORMS IN MAMMALIAN CELLS}

Prx is a family of antioxidant enzymes that removes $\mathrm{H}_{2} \mathrm{O}_{2}$ using the thioredoxin ( $\operatorname{Trx}$ )/Trx reductase/NADPH system as a reducing equivalent [13]. The six isoforms of mammalian Prxs (Prx1-6) possess one or two conserved Cys residues (peroxidatic Cys; $C_{P}$, resolving $C_{y s} ; C_{R}$ ). Prx isoforms are categorized into three different forms depending on the number and position of the conserved Cys residue and on the type of the disulfide bond created during the catalytic cycle. These include typical 2-Cys Prx, atypical 2-Cys Prx, and 1-Cys Prx that are represented by Prx1-4, Prx5, and Prx6, respectively (Fig. 1) [14].

Typical 2-Cys Prxs form a sulfenic acid ( $\left.\mathrm{C}_{\mathrm{P}} \mathrm{SOH}\right)$ intermediate upon the oxidation of $\mathrm{C}_{\mathrm{P}}$-SH by $\mathrm{H}_{2} \mathrm{O}_{2}$, which creates an intermolecular disulfide bond with the $\mathrm{C}_{\mathrm{R}}$ of another Prx subunit in the vicinity. Prx is subsequently reactivated as the disulfide bond is reduced from reducing equivalents [15]. Prx1-4 belong to the typical 2-Cys Prxs class. Prx1 and Prx2 reside mainly in the cytosol in abundance, but they are also found in the nucleus [16]. Prx3 has a mitochondrial leader sequence (MLS) at the N-terminal, directing its localization to the mitochondria [17]. Mitochondria is the major source of ROS, and Prx3 plays a key role in regulating mitochondrial redox homoeostasis [18,19]. Prx4 is found in the endoplasmic reticulum and the extracellular space [20,21]. Atypical 2-Cys Prx uses the same catalytic mechanism as typical 2-Cys Prxs to remove $\mathrm{H}_{2} \mathrm{O}_{2}$, with the only difference being the creation of an intramolecular disulfide bond between $C_{P}$ and $C_{R}$ within a single Prx subunit [22]. Prx5 belongs to the class of atypical 2-Cys Prx. it has a MLS and is largely located in the mitochondria as well as prx3, although it is also found in peroxisomes and the cytosol [17]. Lastly, Prx6 belongs to the 1-Cys Prx class. It is characterized by the lack of $C_{R}$, which is present in other members of the Prx family, and it is expressed in the cytosol [23].

\section{THE PHYSIOLOGICAL ROLE OF PEROXIREDOXINS IN CANCER SIGNALING}

The major enzymatic function of Prxs is to regulate cellular redox signaling as peroxidases, but they also function as molecular chaperones upon $\mathrm{H}_{2} \mathrm{O}_{2}$ concentration-dependent structural changes [24]. Furthermore, in many cancer types, including lung, breast, prostate, and gastric cancers, Prx expression has been known to be high and associated with poor patient prognosis [25-28]. In cancer signaling pathways, Prx has been increasingly reported to either facilitate or inhibit tumorigenesis through its interaction with other signaling proteins in a redox-dependent or redox-independent manner (Table 1) [16,26,28-38]. Among the Prx isoforms, Prx1 and Prx 2 are localized abundantly in the nucleus and the cytosol, which has allowed numerous studies to focus on their expression and their interaction with signaling target proteins in cancer [39,40]. Nonetheless, the relatively low expression of Prx3-6 in cancer and their restricted localization have resulted in a comparatively small number of studies related to cancer.

A

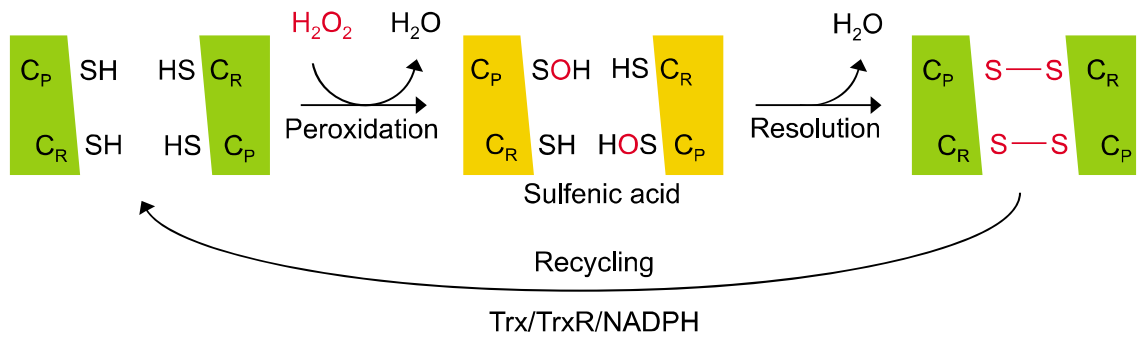

B

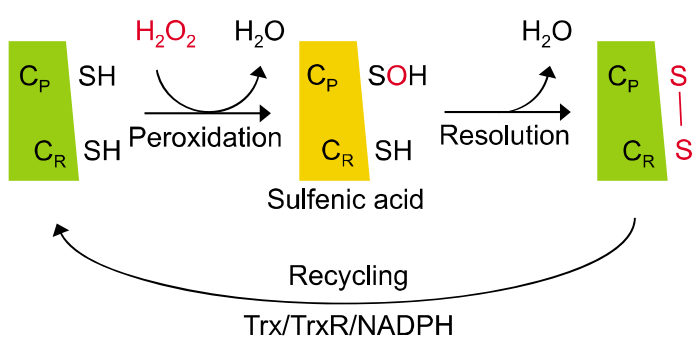

C

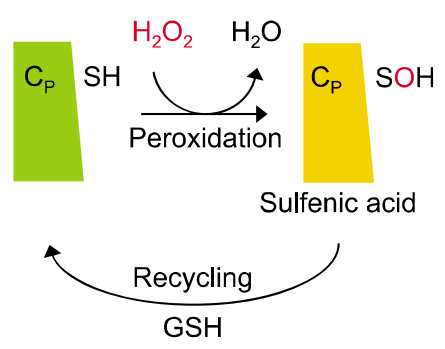

Figure 1. Catalytic cycle of peroxiredoxin (Prx) isoforms. (A) Typical 2-Cys Prxs (Prx1-4) first mediate the oxidation of $C_{P}$ residues to sulfenic acid by $\mathrm{H}_{2} \mathrm{O}_{2}$, followed by the formation of an intermolecular disulfide bond with the $C_{R}$ of another Prx subunit. Lastly, the oxidized Prx undergoes reduction by the thioredoxin $(\operatorname{Tr} \mathrm{x}) / \operatorname{Trx}$ reductase (TrxR)/NADPH system. (B) Atypical 2-Cys Prx (Prx5) is similar to typical 2-Cys Prxs, except that they mediate the formation of an intramolecular disulfide bond with the $C_{R}$ of the same Prx subunit. (C) 1-Cys Prx (Prx6) has only one conserved Cys residue so that they are recycled in sulfenic acid without forming a disulfide bond, which is reduced by glutathione (GSH) instead of Trx. 
Table 1. Expression of Prx isoforms in cancer

\begin{tabular}{ccclc}
\hline Class & Isoform & Expression & Cancer type & Ref. No. \\
\hline Typical 2-Cys & \multirow{2}{*}{ Prx1 } & Up & Lung & {$[29,30]$} \\
& & Up/down & Thyroid & {$[31]$} \\
& & Up & Pancreas & {$[32]$} \\
& & Up & Bladder & {$[33]$} \\
& & Up & Breast & {$[26]$} \\
& & Up & Prostate & {$[34]$} \\
& \multirow{2}{*}{ Prx2 } & Up & Lung & {$[29]$} \\
& & Up & Ovary & {$[35]$} \\
& \multirow{2}{*}{ Prx3 } & Up & Breast & {$[26,36]$} \\
& & Up & Cervix & {$[37]$} \\
& & Up & Prostate & {$[38]$} \\
& \multirow{2}{*}{ Prx4 } & Up & Lung & {$[29]$} \\
& & Up & Breast & {$[26]$} \\
Atypical 2-Cys & \multirow{2}{*}{ Prx5 } & Up & Prostate & {$[38]$} \\
& & Up & Breast & {$[26]$} \\
1-Cys & \multirow{2}{*}{ Prx6 } & Up & Stomach & {$[28]$} \\
& & Up & Lung & {$[29]$} \\
& & Up & Bladder & {$[33]$} \\
& & & & {$[35]$} \\
\hline
\end{tabular}

Prx, peroxiredoxin.

\section{Prx1}

Smoking, one of the multiple causes of malignant lung cancer, results in $80 \%$ to $90 \%$ of lung cancer deaths [41]. Numerous therapies, including radiation therapy (radiotherapy), have been developed and used to treat lung cancer and many other types of malignancies [42]. Radiation therapy increases the level of ROS to facilitate apoptosis in cancer cells [43,44]. Prxl is highly expressed in several human cancers, including lung cancer. Prx1 enhances lung cancer cell survival by suppressing radiationinduced apoptosis $[29,30]$. Glutathione- $S$ Transferase Pi (GSTP) is a protein that binding to the c-Jun N-terminal kinase (JNK) to prevent its activation, thereby inhibiting apoptosis. Radiation dissociates the interaction between the two proteins, and the released JNK is activated to promote apoptosis. However, Prx1 overexpression increases the binding of the GSTP-JNK complex independently of its peroxidase activity. Consequently, Prx1 inhibits the release of JNK from GSTP, thereby suppressing the radiation-induced JNK activation in lung cancer and preventing apoptosis [45].

Prostate cancer ( $\mathrm{PCa}$ ) is the most common malignancy in males [46]. Dihydrotestosterone (DHT), which is converted form androgen testosterone by $5 \alpha$-reductase, binds to the androgen receptor (AR) in the cytosol, and the androgen/AR complex enters the nucleus to bind to androgen response element (ARE) on the target gene promoter. The resulting increase in expression of the downstream target genes such as prostate specific antigen (PSA) promotes the progression of PCa [47]. Hormone therapies, the androgen deprivation therapy (ADT), are commonly used treatment, in which inhibitors of androgen production or AR are used. However, once PCa develops resistance to $\mathrm{ADT}$, the condition becomes castration-resistant $\mathrm{PCa}$, rendering hormone therapy ineffective [48]. The expression of Prx1 is highly in prostate cancer cells [34]. The DHT binding affinity of AR is increased by Prxl which is independent of its peroxidase activity, and this promotes androgen-stimulated prostate cancer growth. Furthermore, in hypoxia, Prxl binds to AR independently of its peroxidase activity, and this increases the binding affinity of $A R$ to the ARE of the PSA promoter to increase ligand-stimulated AR activation [49].

The PI3K/AKT signaling pathway is crucial for cancer survival because it facilitates tumorigenesis through increased cell growth and proliferation [50,51]. In this pathway, when a growth factor binds to the receptor to activate the receptor-bound PI3K, the activated PI3K phosphorylates the precursor $\mathrm{PIP}_{2}$ to convert it to $\mathrm{PIP}_{3}$ as a mediator. $\mathrm{PIP}_{3}$ activates AKT phosphorylation by PDK1 to regulate various downstream cancer-associated signaling pathways [52]. Furthermore PTEN is a tumor suppressor protein that uses its lipid phosphatase activity to dephosphorylate $\mathrm{PIP}_{3}$ and convert it to $\mathrm{PIP}_{2}$, which subsequently prevents AKT activation and consequently the activation of the PI3K/AKT signaling pathway [53]. However, oxidation of PTEN caused by oxidative stress leads to its inactivation. Prx1 binds to PTEN to prevent its oxidation, thereby inhibiting tumorigenesis in breast cancer [12].

\section{2. $\operatorname{Prx} 2$}

DNA methylation, which induces gene silencing, can occur on the promoters of tumor suppressors, increasing the growth of cancer cells [54]. DNA methyltransferases (DNMTs), enzymes essential for DNA methylation, catalyze the transfer of a methyl group to the CpG structure in the DNA. DNMTs include DNMT1, DNMT3A and DNMT3B [55]. This methylation occurs in the Prx2 gene promoter in gastric cancer, lymphoma, and melanoma. Unlike Prx1, Prx2 shows decreased mRNA and protein expression in approximately 32\% (9 out of 28) of gastric cancer cell lines. DNMT1 suppresses Prx 2 expression by promoting the methylation of Prx2. Contrarily, increased Prx2 expression in gastric cancer suppresses Src kinase activation through its peroxidase activity to inhibit the survival and migration of gastric cancer cells [56].

In patients with metastatic disease, the 5 -year survival rate is less than $15 \%$, indicating that tumor metastasis is a considerable 
threat to the patient's survival [57]. As a highly metastatic cancer, melanoma is the deadliest skin cancer. $\mathrm{H}_{2} \mathrm{O}_{2}$ increases the ERK and Src activation, and these in turn suppress the expression of E-cadherin and promote the phosphorylation of $\beta$-catenin, respectively. This promotes the dissociation of E-cadherin/ $\beta$-catenin complexes to cause epithelial-to-mesenchymal transition (EMT). As a result, cells metastasize to the lung or other organs. However, Prx2 removes $\mathrm{H}_{2} \mathrm{O}_{2}$ in melanoma cells, thereby increasing E-cadherin expression by suppressing ERK and Src activities. Additionally, the removal of $\mathrm{H}_{2} \mathrm{O}_{2}$ increases the membrane retention of $\beta$-catenin by suppressing Src-mediated $\beta$-catenin phosphorylation. The peroxidase-inactive (C51/172S) Prx2 mutant fails to remove $\mathrm{H}_{2} \mathrm{O}_{2}$, leading to melanoma metastasis. These results suggest that the peroxidase activity of $\operatorname{Prx} 2$ is essential to prevent metastasis. Thus, $\operatorname{Prx} 2$ removes $\mathrm{H}_{2} \mathrm{O}_{2}$ in melanoma cells to promote the E-cadherin/ $\beta$-catenin complexes, preventing metastasis [58].

\section{Prx3}

Prx3 is mainly localized to the mitochondria, and is highly expressed in various cancer types including PCa, breast cancer, and hepatocellular carcinoma [26,36,59,60]. In PCa, non-steroidal anti-androgen bicalutamide antagonizes the binding between androgen and $\mathrm{AR}$, and is therefore used in anti-androgen treatment. However, PCa gradually develops resistance to this treatment. Anti-androgen resistant cells contain an increased number of mitochondria, with a consequent increase in mitochondrial Prx3 expression. The increased level of Prx3 protects the mitochondria from $\mathrm{H}_{2} \mathrm{O}_{2}$-induced oxidative stress, thereby inhibiting apoptosis in PCa [61].

\section{Prx4}

Sulfiredoxin (Srx) is a protein that reversibly reduces the Cys- $\mathrm{SO}_{2} \mathrm{H}$ form of hyperoxidized Prx. The peroxidase activity of the reactivated Prx through the Srx-mediated Prx reversible reaction, protects the cells from oxidative stress [62]. However, Srx is highly expressed in lung cancer, where it increases cancer cell proliferation and invasion [63]. Among the typical 2-Cys Prxs, Prx4 exhibits the strongest binding affinity upon interaction with Srx, and similar to Srx, Prx4 shows a high level of expression in lung cancer, where it promotes cancer cell proliferation [29]. Such facilitation of tumorigenesis via the Srx-Prx 4 axis is regulated by two pathways. In the first pathway, c-Jun phosphorylation is increased by Srx, resulting in activation of the transcription factor AP-1 and increased expression of the downstream target MMP9, which lead to the regulation of Srx-Prx4. In the second pathway, this regulation relies on an Srx-dependent increase of ERK1/2 phosphorylation. Srx activates the phosphorylation of c-Jun, a transcription factor activator protein (AP-1, a heterodimer of c-Fos and c-Jun), to increase MMP9 expression, which promotes the invasion and metastasis of lung cancer. Srx also facilitates lung cancer proliferation by increasing the phosphorylation of ERK1/2 and CREB. Likewise, Prx4 activates the phosphorylation of ERK1/2, AKT, CREB, and c-Jun. Therefore, the Srx-Prx4 axis plays a role in promoting tumor growth and metastasis of lung cancer [64].

\section{Prx5}

EMT is a cellular process that promotes cell proliferation, invasion, and metastasis in various cancer types [65]. ROS facilitates gastric and lung cancer cell migration and invasion [66,67]. Unlike Prx1-4, a high level of Prx5 expression in patients with gastric cancer is associated with a markedly reduced 5-year rate of survival. Prx5 increases vimentin expression and decreases E-cadherin expression in gastric cancer cells, thereby promoting not only EMT, but also tumorigenesis through increased invasion and proliferation [28].

\section{Prx6}

Prx6 differs from other Prx isoforms as it contains only one conserved Cys (C47), but it is highly expressed in lung cancer, similar to others [23]. As Prx6 exhibits calcium-independent phospholipase A2 (iPLA2) and glutathione peroxidase (GPx) activities, an increase in Prx6 leads to elevated activities of iPLA2 and GPx. The iPLA2 and GPx activities are used by Prx6 to promote the expression of cell cycle regulatory proteins (CDK1, CDK2, and cyclin D1). Moreover, by activating MAP kinase pathway proteins (ERK1/2 and p38), Prx6 enhances the DNA binding activity of AP-1, which consequently promotes the growth and viability of lung cancer cells. A mutant form of Prx6 (C47S) lacking the iPLA2 and GPx activities suppresses the p38 and ERK1/2 activation and DNA binding activity of AP-1, consequently decreasing tumorigenesis. Prx6 thus facilitates tumorigenesis in lung cancer through its peroxidase activity [68].

\section{CONCLUSION}

Prxs exert their key function of regulation of redox homeostasis through their antioxidant enzymatic activity, which removes intracellular $\mathrm{H}_{2} \mathrm{O}_{2}$. The $6 \mathrm{Prx}$ isoforms act as $\mathrm{H}_{2} \mathrm{O}_{2}$ scavengers in different intracellular compartments. Additionally, at high $\mathrm{H}_{2} \mathrm{O}_{2}$ concentrations, Prxs become molecular chaperones 
through gain of function. Thus, Prxs are multifunctional proteins which play different roles. Recently, a growing number of studies have found that Prxs regulate their expression and activity, acting as oncogenes that promote carcinogenesis in various cancer types. In addition, Prxs are known to promote the cancer cell stemness [69-71]. Nevertheless, as Prxs have also been shown to suppress tumorigenesis, more studies are required to verify the potential of Prxs as therapeutic targets in each cancer type.

Furthermore, Prxs influence the progression of diseases including cancer, depending on their protein-protein interactions. Among the Prx isoforms, Prx 1 and 2 are the most abundant, and hence their interaction partners have been more extensively studied. In addition, to investigate the possibility that each Prx isoform mediates a different mechanism of cancer signaling regulation as they are located in different intracellular organelles, further studies should focus on the identification of interaction partners of each isoform and the elucidation of underlying mechanisms. In conclusion, the potential application of Prxs as therapeutic strategies for cancer treatment is promising.

\section{ACKNOWLEDGMENTS}

This research was supported by the Basic Science Research Program through the National Research Foundation of Korea (NRF) funded by the Ministry of Education (NRF-2018R1A2B6 009042).

\section{CONFLICTS OF INTEREST}

No potential conflicts of interest were disclosed.

\section{REFERENCES}

1. Dagogo-Jack I, Shaw AT. Tumour heterogeneity and resistance to cancer therapies. Nat Rev Clin Oncol 2018;15:81-94.

2. Bhattacharyya A, Chattopadhyay R, Mitra S, Crowe SE. Oxidative stress: an essential factor in the pathogenesis of gastrointestinal mucosal diseases. Physiol Rev 2014:94:329-54.

3. Waris G, Ahsan H. Reactive oxygen species: role in the development of cancer and various chronic conditions. J Carcinog 2006; 5:14.

4. Nimse SB, Pal D. Free radicals, natural antioxidants, and their reaction mechanisms. RSC Adv 2015:5:27986-8006.

5. Birben E, Sahiner UM, Sackesen C, Erzurum S, Kalayci O. Oxidative stress and antioxidant defense. World Allergy Organ J 2012;5: 9-19.

6. Rhee SG, Chae HZ, Kim K. Peroxiredoxins: a historical overview and speculative preview of novel mechanisms and emerging concepts in cell signaling. Free Radic Biol Med 2005:38:1543-52.

7. Rhee SG, Kil IS. Multiple functions and regulation of mammalian peroxiredoxins. Annu Rev Biochem 2017;86:749-75.

8. Nicolussi A, D'Inzeo S, Capalbo C, Giannini G, Coppa A. The role of peroxiredoxins in cancer. Mol Clin Oncol 2017;6:139-53.

9. Luo W, Chen I, Chen Y, Alkam D, Wang Y, Semenza GL. PRDX2 and PRDX4 are negative regulators of hypoxia-inducible factors under conditions of prolonged hypoxia. Oncotarget 2016;7: 6379-97.

10. Park SY, Yu X, Ip C, Mohler JL, Bogner PN, Park YM. Peroxiredoxin 1 interacts with androgen receptor and enhances its transactivation. Cancer Res 2007;67:9294-303.

11. Kang DH, Lee DJ, Lee S, Lee SY, Jun Y, Kim Y, et al. Interaction of tankyrase and peroxiredoxin II is indispensable for the survival of colorectal cancer cells. Nat Commun 2017;8:40.

12. Cao J, Schulte J, Knight A, Leslie NR, Zagozdzon A, Bronson R, et al. Prdx1 inhibits tumorigenesis via regulating PTEN/AKT activity. EMBO J 2009;28:1505-17.

13. Rhee SG, Woo HA. Multiple functions of peroxiredoxins: peroxidases, sensors and regulators of the intracellular messenger $\mathrm{H}_{2} \mathrm{O}_{2}$, and protein chaperones. Antioxid Redox Signal 2011; 15:781-94.

14. Rhee SG, Woo HA, Kil IS, Bae SH. Peroxiredoxin functions as a peroxidase and a regulator and sensor of local peroxides. J Biol Chem 2012;287:4403-10.

15. Hall A, Karplus PA, Poole LB. Typical 2-Cys peroxiredoxins--structures, mechanisms and functions. FEBS J 2009;276: 2469-77.

16. Park J, Lee S, Lee S, Kang SW. 2-cys peroxiredoxins: emerging hubs determining redox dependency of Mammalian signaling networks. Int J Cell Biol 2014:2014:715867.

17. Cox AG, Winterbourn CC, Hampton MB. Mitochondrial peroxiredoxin involvement in antioxidant defence and redox signalling. Biochem J 2009:425:313-25.

18. Cunniff B, Wozniak AN, Sweeney P, DeCosta K, Heintz NH. Peroxiredoxin 3 levels regulate a mitochondrial redox setpoint in malignant mesothelioma cells. Redox Biol 2014;3:79-87.

19. Murphy MP. How mitochondria produce reactive oxygen species. Biochem J 2009;417:1-13.

20. Tavender TJ, Sheppard AM, Bulleid NJ. Peroxiredoxin IV is an endoplasmic reticulum-localized enzyme forming oligomeric complexes in human cells. Biochem J 2008:411:191-9.

21. Schulte J. Peroxiredoxin 4: a multifunctional biomarker worthy of further exploration. BMC Med 2011;9:137.

22. Seo MS, Kang SW, Kim K, Baines IC, Lee TH, Rhee SG. Identification of a new type of mammalian peroxiredoxin that forms an intramolecular disulfide as a reaction intermediate. J Biol Chem 2000;275:20346-54.

23. Manevich $Y$, Fisher AB. Peroxiredoxin 6, a 1-Cys peroxiredoxin, functions in antioxidant defense and lung phospholipid metabolism. Free Radic Biol Med 2005:38:1422-32.

24. Veal EA, Underwood ZE, Tomalin LE, Morgan BA, Pillay CS. Hyperoxidation of peroxiredoxins: gain or loss of function? Antioxid Redox Signal 2018;28:574-90.

25. Park JH, Kim YS, Lee HL, Shim JY, Lee KS, Oh YJ, et al. Expression of peroxiredoxin and thioredoxin in human lung cancer and paired normal lung. Respirology 2006;11:269-75.

26. Karihtala P, Mäntyniemi A, Kang SW, Kinnula VL, Soini Y. Peroxiredoxins in breast carcinoma. Clin Cancer Res 2003;9: 3418-24.

27. Chaiswing L, Zhong W, Oberley TD. Increasing discordant anti- 
oxidant protein levels and enzymatic activities contribute to increasing redox imbalance observed during human prostate cancer progression. Free Radic Biol Med 2014:67:342-52.

28. Kim B, Kim YS, Ahn HM, Lee HJ, Jung MK, Jeong HY, et al. Peroxiredoxin 5 overexpression enhances tumorigenicity and correlates with poor prognosis in gastric cancer. Int J Oncol 2017; 51:298-306.

29. Lehtonen ST, Svensk AM, Soini Y, Pääkkö P, Hirvikoski P, Kang SW, et al. Peroxiredoxins, a novel protein family in lung cancer. Int J Cancer 2004;111:514-21.

30. Chen L, Huang C, Yang X, Zhang Q, Chen F. Prognostic roles of mRNA expression of peroxiredoxins in lung cancer. Onco Targets Ther 2018;11:8381-8.

31. Yanagawa $\mathrm{T}$, Ishikawa $\mathrm{T}$, Ishii $\mathrm{T}$, Tabuchi $\mathrm{K}$, Iwasa $\mathrm{S}$, Bannai $\mathrm{S}$, et al. Peroxiredoxin I expression in human thyroid tumors. Cancer Lett 1999;145:127-32.

32. Cai CY, Zhai LL, Wu Y, Tang ZG. Expression and clinical value of peroxiredoxin-1 in patients with pancreatic cancer. Eur J Surg Oncol 2015:41:228-35.

33. Quan C, Cha EJ, Lee HL, Han KH, Lee KM, Kim WJ. Enhanced expression of peroxiredoxin I and VI correlates with development, recurrence and progression of human bladder cancer. J Urol 2006; 175:1512-6.

34. Riddell JR, Bshara W, Moser MT, Spernyak JA, Foster BA, Gollnick SO. Peroxiredoxin 1 controls prostate cancer growth through Toll-like receptor 4-dependent regulation of tumor vasculature. Cancer Res 2011;71:1637-46.

35. Pylväs M, Puistola U, Kauppila S, Soini Y, Karihtala P. Oxidative stress-induced antioxidant enzyme expression is an early phenomenon in ovarian carcinogenesis. Eur J Cancer 2010;46: 1661-7.

36. Chua PJ, Lee EH, Yu Y, Yip GW, Tan PH, Bay BH. Silencing the Peroxiredoxin III gene inhibits cell proliferation in breast cancer. Int J Oncol 2010;36:359-64.

37. Hu JX, Gao Q, Li L. Peroxiredoxin 3 is a novel marker for cell proliferation in cervical cancer. Biomed Rep 2013;1:228-30.

38. $\mathrm{Li} \mathrm{L}, \mathrm{Yu} \mathrm{AQ}$. The functional role of peroxiredoxin 3 in reactive oxygen species, apoptosis, and chemoresistance of cancer cells. J Cancer Res Clin Oncol 2015;141:2071-7.

39. Bertoldi M. Human peroxiredoxins 1 and 2 and their interacting protein partners: through structure toward functions of biological complexes. Protein Pept Lett 2016;23:69-77.

40. Lee W, Choi KS, Riddell J, Ip C, Ghosh D, Park JH, et al. Human peroxiredoxin 1 and 2 are not duplicate proteins: the unique presence of CYS83 in Prx1 underscores the structural and functional differences between Prx1 and Prx2. J Biol Chem 2007; 282:22011-22.

41. Goldkorn T, Filosto S, Chung S. Lung injury and lung cancer caused by cigarette smoke-induced oxidative stress: molecular mechanisms and therapeutic opportunities involving the ceramide-generating machinery and epidermal growth factor receptor. Antioxid Redox Signal 2014;21:2149-74.

42. Baskar R, Lee KA, Yeo R, Yeoh KW. Cancer and radiation therapy: current advances and future directions. Int J Med Sci 2012;9: 193-9.

43. Wang J, Yi J. Cancer cell killing via ROS: to increase or decrease, that is the question. Cancer Biol Ther 2008;7:1875-84.

44. O'Connor MJ. Targeting the DNA damage response in cancer. Mol Cell 2015;60:547-60.
45. Kim YJ, Lee WS, Ip C, Chae HZ, Park EM, Park YM. Prx1 suppresses radiation-induced c-Jun NH2-terminal kinase signaling in lung cancer cells through interaction with the glutathione S-transferase Pi/c-Jun NH2-terminal kinase complex. Cancer Res 2006; 66:7136-42.

46. Velonas VM, Woo HH, dos Remedios CG, Assinder SJ. Current status of biomarkers for prostate cancer. Int J Mol Sci 2013;14: $11034-60$.

47. Feldman BJ, Feldman D. The development of androgen-independent prostate cancer. Nat Rev Cancer 2001;1:34-45.

48. Yuan X, Cai C, Chen S, Chen S, Yu Z, Balk SP. Androgen receptor functions in castration-resistant prostate cancer and mechanisms of resistance to new agents targeting the androgen axis. Oncogene 2014:33:2815-25.

49. Chhipa RR, Lee KS, Onate S, Wu Y, Ip C. Prxl enhances androgen receptor function in prostate cancer cells by increasing receptor affinity to dihydrotestosterone. Mol Cancer Res 2009;7:1543-52.

50. Davis WJ, Lehmann PZ, Li W. Nuclear PI3K signaling in cell growth and tumorigenesis. Front Cell Dev Biol 2015;3:24.

51. Testa JR, Bellacosa A. AKT plays a central role in tumorigenesis. Proc Natl Acad Sci U S A 2001;98:10983-5.

52. Jiang BH, Liu LZ. PI3K/PTEN signaling in angiogenesis and tumorigenesis. Adv Cancer Res 2009;102:19-65.

53. Yamada KM, Araki M. Tumor suppressor PTEN: modulator of cell signaling, growth, migration and apoptosis. J Cell Sci 2001;114(Pt 13):2375-82.

54. Raynal NJ, Si J, Taby RF, Gharibyan V, Ahmed S, Jelinek J, et al. DNA methylation does not stably lock gene expression but instead serves as a molecular mark for gene silencing memory. Cancer Res 2012;72:1170-81.

55. Gujar H, Weisenberger DJ, Liang G. The roles of human DNA methyltransferases and their isoforms in shaping the epigenome. Genes (Basel) 2019;10:E172.

56. Hong SH, Min C, Jun Y, Lee DJ, Kim SH, Park JH, et al. Silencing of peroxiredoxin II by promoter methylation is necessary for the survival and migration of gastric cancer cells. Exp Mol Med 2018:50:e443.

57. Sandru A, Voinea S, Panaitescu E, Blidaru A. Survival rates of patients with metastatic malignant melanoma. J Med Life 2014;7: 572-6.

58. Lee DJ, Kang DH, Choi M, Choi YJ, Lee JY, Park JH, et al. Peroxiredoxin-2 represses melanoma metastasis by increasing E-Cadherin/ $\beta$-Catenin complexes in adherens junctions. Cancer Res 2013;73:4744-57.

59. Newick K, Cunniff B, Preston K, Held P, Arbiser J, Pass H, et al. Peroxiredoxin 3 is a redox-dependent target of thiostrepton in malignant mesothelioma cells. PLoS One 2012;7:e39404.

60. Basu A, Banerjee H, Rojas H, Martinez SR, Roy S, Jia Z, et al. Differential expression of peroxiredoxins in prostate cancer: consistent upregulation of PRDX3 and PRDX4. Prostate 2011;71: $755-65$.

61. Whitaker HC, Patel D, Howat WJ, Warren AY, Kay JD, Sangan T, et al. Peroxiredoxin-3 is overexpressed in prostate cancer and promotes cancer cell survival by protecting cells from oxidative stress. Br J Cancer 2013;109:983-93.

62. Rhee SG, Jeong W, Chang TS, Woo HA. Sulfiredoxin, the cysteine sulfinic acid reductase specific to 2-Cys peroxiredoxin: its discovery, mechanism of action, and biological significance. Kidney Int Suppl 2007;(106):S3-8. 
63. Kim YS, Lee HL, Lee KB, Park JH, Chung WY, Lee KS, et al. Nuclear factor E2-related factor 2 dependent overexpression of sulfiredoxin and peroxiredoxin III in human lung cancer. Korean J Intern Med 2011:26:304-13.

64. Wei $\mathrm{Q}$, Jiang $\mathrm{H}$, Xiao Z, Baker A, Young MR, Veenstra TD, et al. Sulfiredoxin-Peroxiredoxin IV axis promotes human lung cancer progression through modulation of specific phosphokinase signaling. Proc Natl Acad Sci U S A 2011;108:7004-9.

65. Pastushenko I, Blanpain C. EMT transition states during tumor progression and metastasis. Trends Cell Biol 2019;29:212-26.

66. Luanpitpong S, Talbott SJ, Rojanasakul Y, Nimmannit U, Pongrakhananon V, Wang L, et al. Regulation of lung cancer cell migration and invasion by reactive oxygen species and caveolin-1. J Biol Chem 2010;285:38832-40.

67. Zhao W, Lu M, Zhang Q. Chloride intracellular channel 1 regulates migration and invasion in gastric cancer by triggering the
ROS-mediated p38 MAPK signaling pathway. Mol Med Rep 2016;13:3711

68. Yun HM, Park KR, Lee HP, Lee DH, Jo M, Shin DH, et al. PRDX6 promotes lung tumor progression via its GPx and iPLA2 activities. Free Radic Biol Med 2014;69:367-76.

69. Song IS, Jeong YJ, Seo YJ, Byun JM, Kim YN, Jeong DH, et al. Peroxiredoxin 3 maintains the survival of endometrial cancer stem cells by regulating oxidative stress. Oncotarget 2017:8: 92788-800.

70. Son YW, Cheon MG, Kim Y, Jang HH. Prx2 links ROS homeostasis to stemness of cancer stem cells. Free Radic Biol Med 2019;134: 260-7.

71. Chandimali N, Jeong DK, Kwon T. Peroxiredoxin II regulates cancer stem cells and stemness-associated properties of cancers. Cancers (Basel) 2018;10:E305. 